\title{
A Case of Localized Pullmonary Calcification Presenting as a Persistent Mass Lesion in an Immunosuppressed Patient Following Treatment of a Pseudomonas Pneumonia
}

\author{
Matthew J. Cecchini ${ }^{1}$, Dominic L. Shepherd ${ }^{2}$, Jessica G. Shepherd ${ }^{3}$ \\ 1. Department of Pathology, Schulich School of Medicine \& Dentistry, Western University, London, \\ Ontario, CA 2. Schulich School of Medicine \& Dentistry, Western University, London, Ontario, CA 3. \\ Department of Pathology and Laboratory Medicine, London Health Science Centre
}

$\square$ Corresponding author: Matthew J. Cecchini, matt.cecchini@lhsc.on.ca Disclosures can be found in Additional Information at the end of the article

\section{Abstract}

We report a case of a persistent right upper lobe opacity following treatment for a Pseudomonas infection in an immunosuppressed patient with a recent renal transplantation. The patient underwent a surgical lung biopsy for definitive diagnosis of the mass. The lesion was composed of extensive calcifications deposited throughout the lung with associated fibrosis. The patient had a history of a remote parathyroidectomy for hyperparathyroidism; however, the parathyroid hormone (PTH) and the calcium levels were still mildly elevated. No other calcified lung lesions had developed in a follow-up after the initial resection. Pulmonary calcification has been classically associated with varicella pneumonia; no viral cytopathic changes were identified for varicella or other viruses in this case. The calcification appears to be secondary to the recent Pseudomonas pneumonia. To our knowledge, this is the first report of a Pseudomonas pneumonia resulting in extensive localized pulmonary calcification. This is an important diagnostic consideration as this benign entity should be considered in patients with persistent opacities following treatment for pneumonia.

Categories: Pathology

Keywords: pulmonary calcification, immunosupressed, pseudomonas

\section{Introduction}

Received 08/28/2017 Review began 09/08/2017 Review ended 10/08/2017 Published 10/11/2017

\section{(c) Copyright 2017}

Cecchini et al. This is an open access article distributed under the terms of the Creative Commons Attribution License CC-BY 3.0., which permits unrestricted use, distribution, and reproduction in any medium, provided the original author and source are credited.
Parenchymal pulmonary calcification can be separated into dystrophic and metastatic calcifications. Dystrophic calcification occurs in damaged lungs typically following an inflammatory process as a localized organized collection of hydroxyapatite calcium salt [1]. Metastatic pulmonary calcification is caused by high serum calcium and phosphate levels that accumulate in normal lung tissue. The elevated calcium is usually from renal failure, hyperparathyroidism or neoplastic destruction of bone [1]. Calcium deposition favours alkaline tissues, in particular in the upper lobes of the lungs, which have a high ventilation to perfusion $(\mathrm{V} / \mathrm{Q})$ ratio and low end capillary $\mathrm{pCO}_{2}$, resulting in a more alkaline $\mathrm{pH}$ [2-3]. Pulmonary calcifications are typically incidental findings identified on imaging and rarely result in significant respiratory disease [1].

We report a case of a patient presenting with a persistent right upper lobe mass following a 


\section{Cureus}

Pseudomonas pneumonia. There was a history of chronic kidney disease with renal transplantation and a remote history of a parathyroidectomy with residual mildly elevated parathyroid hormone (PTH) and calcium levels.

\section{Case Presentation}

The patient is a female in her 40s who underwent a repeat renal transplantation that was complicated by acute tubular necrosis, delayed graft function, and Pseudomonas sepsis with a right upper lobe pneumonia (Figure $1 \mathrm{~A}$ ). The patient was admitted and treated with an extended course of piperacillin-tazobactam for the Pseudomonas infection. She continued to experience dyspnea following the completion of treatment and had a persistent right upper lobe opacity identified on chest X-ray (Figure $1 B$ ) and the subsequent computed tomography (CT) scan (Figures $2 A-2 B)$. CT scans demonstrated a large opacity $(10 \mathrm{~cm} \mathrm{x} 4 \mathrm{~cm})$ with adjacent areas of mixed ground glass opacity, airspace opacity, and thickening of the interlobular septa. The combination of the ground-glass attenuation with superimposed interlobular septal thickening and reticular thickening-specific opacifications was consistent with a "crazy paving" pattern. Given the history of immunosuppression, infectious, inflammatory, and neoplastic lesions were considered in the differential. A follow-up CT scan two months later demonstrated a persistent lesion with little change from the previous imaging. Given the failure of this lesion to resolve, the patient underwent a surgical lung biopsy to exclude an atypical infectious or neoplastic lesion.
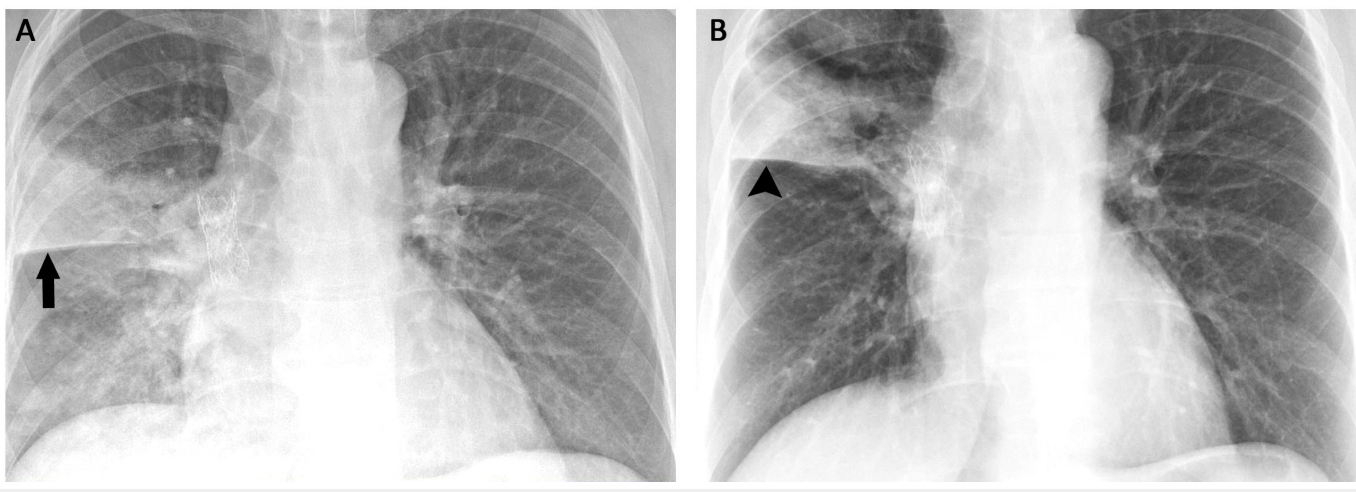

FIGURE 1: (A) Chest radiograph demonstrating the right upper lobe pneumonia (arrow) and (B) persistent right upper lobe opacity (arrowhead) following treatment of the pneumonia.
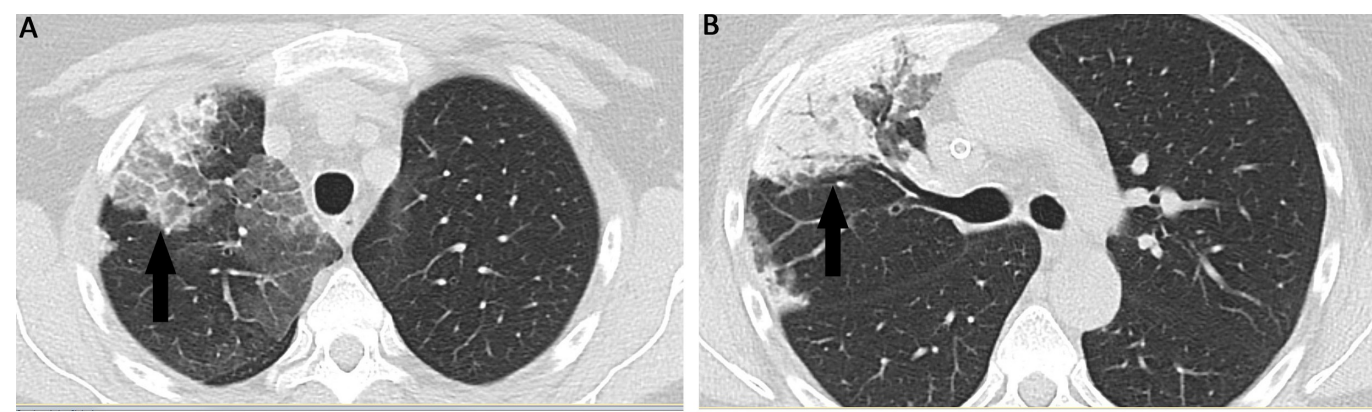

FIGURE 2: (A, B) Representative CT slices showing the right upper lobe opacity (arrow).

CT - computed tomography. 
A video-assisted thoracoscopic surgery (VATS) was performed and an abnormal right upper lobe with a nodular and cheesy appearance was identified. A representative wedge biopsy was sent for intraoperative consultation. The cryosections showed interstitial pneumonitis with calcification and no evidence of malignancy. Given that this appeared to be a benign process, a lobectomy was not performed, but additional wedge biopsies were sent for permanent sections along with material for culture. As shown in Figures 3A, 3C, 3E, 3G, sections of the lung biopsy were composed of extensive multifocal calcifications, highlighted by a von Kossa stain (Figures $3 B, 3 D, 3 F, 3 H)$. The calcifications ranged in size from small stippled foci to larger irregular deposits with scattered foreign body giant cell reactions. The calcifications were localized within the alveolar septa and were associated with collagenous fibrosis and focal fibroblastic proliferation (Figures 3G-3H). Many of the small blood vessels showed marked subendothelial myxoid intimal thickening with associated calcifications (Figures 3E-3F). In contrast, the larger veins and arteries were relatively spared from calcification. The cultures were negative and Grocott's methenamine silver stain (GMS) and Ziehl-Neelsen (ZN) stains on tissue sections were negative for fungal organisms and acid-fast bacteria, respectively. 


\section{Cureus}

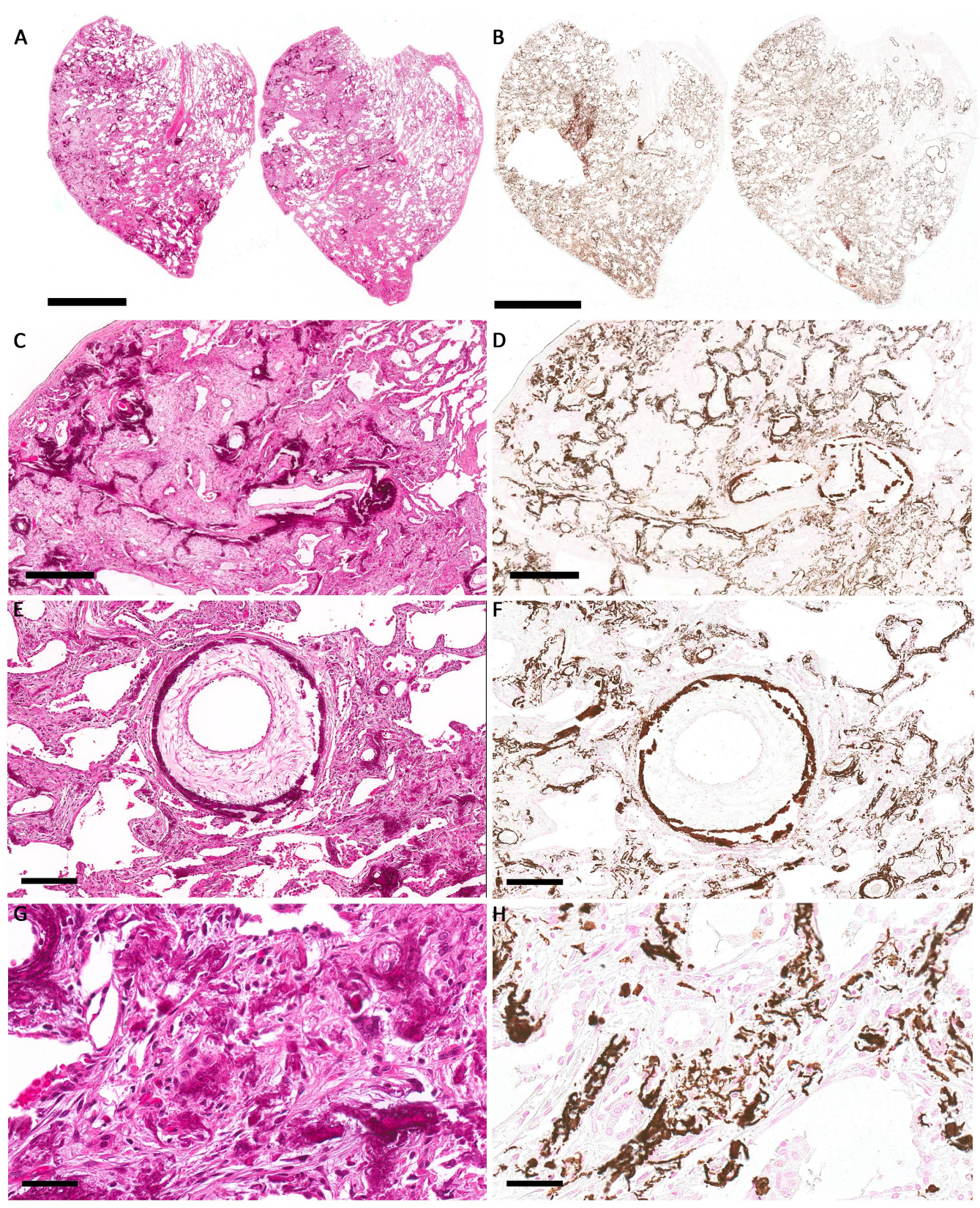

FIGURE 3: Photomicrographs of hematoxylin and eosin stain sections of the wedge biopsy (A, C, E, G) with corresponding areas stained with von Kossa histochemical stain (B, D, G, H) to highlight calcium deposition. (A, B) scale bar $=3 \mathrm{~mm}$, (C, D) scale bar $=\mathbf{5 0 0}$ microns, $(E, F)$ scale bar $=200$ microns, $(G, H)$ scale bar $=100$ microns.

Following the biopsy, the PTH was found to be mildly elevated at $8.1 \mathrm{pmol} / \mathrm{L}$ and associated with a slightly elevated calcium of up to $2.7 \mathrm{mmol} / \mathrm{L}$. In a follow-up six months after the resection, the patient had normal pulmonary function testing, and the X-ray shown in Figure 4 showed a significantly reduced opacity in the right upper lobe of the lung with no new 


\section{Cureus}

consolidations or lesions.

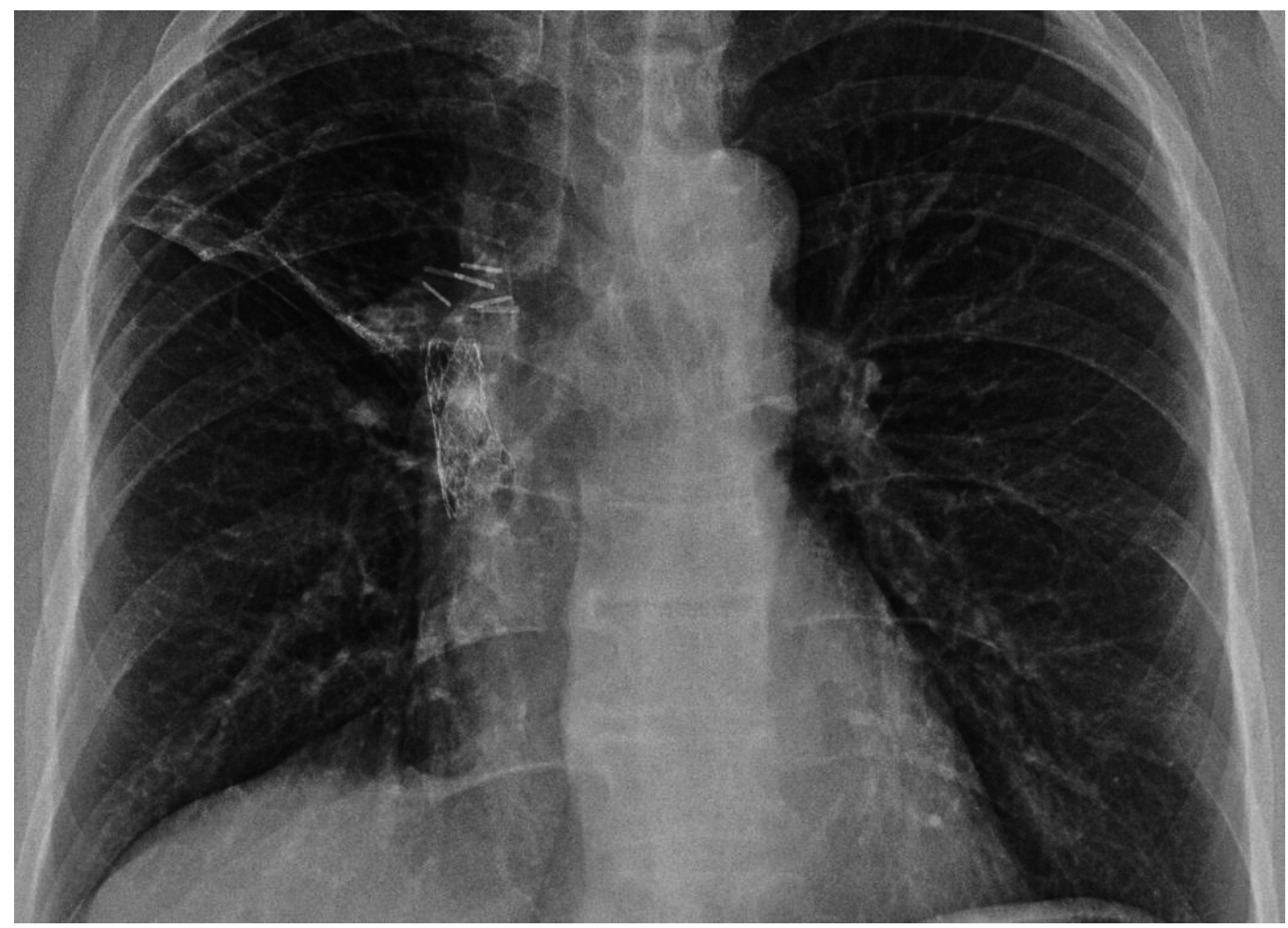

FIGURE 4: Follow up chest X-ray after surgical lung biopsy showing resolution of the right upper lobe opacity.

\section{Discussion}

Pulmonary calcification is typically divided into dystrophic and metastatic calcification. This case represents an overlap of dystrophic and metastatic calcification. While the patient had an elevated serum calcium level, they also had a right upper lobe Pseudomonas pneumonia that may have acted as a nidus for a dystrophic type of calcification. Varicella pneumonia has been classically associated with pulmonary calcification [4]. There was no history of recent varicella infection and no viral cytopathic effects were identified on biopsy. Pulmonary calcification after resolution of other infections have been reported, including Pneumocystis and small pox [4-5]. However, to our knowledge there are no reports of pulmonary calcification following resolution of a Pseudomonas infection. Pulmonary calcifications can also be seen in patients with chronic kidney disease on dialysis [6]; however, these calcifications tend to be more diffuse in nature.

Prominent myxoid intimal thickening was observed in many of the calcified smaller vessels within the biopsy (Figures $3 E-3 F$ ). This has been described as one of the earlier histologic features of pulmonary veno-occlusive disease [7], and recent infection has been suggested to be a potential causative agent [8]. Further, localized pulmonary calcification has been reported in association with vascular obstruction [9]. The mechanism is thought to involve a ventilation perfusion mismatch, where $\mathrm{CO}_{2}$ is still expelled via ventilation but the decreased perfusion results in a decreased supply of $\mathrm{CO}_{2}$ and an increase in the $\mathrm{pH}$. This more alkaline $\mathrm{pH}$ can promote the deposition of calcium into the lung parenchyma.

While pulmonary calcifications tend to have a benign course, there are a number of renal 
transplantation cases with extensive pulmonary calcification that have resulted in acute respiratory failure and even death in isolated cases [10]. These cases have all been associated with kidney graft failure, and improvement in calcification has been seen with improvement of graft function and treatment of underlying hyperparathyroidism [10]. In this case, the patient had a transient acute tubular necrosis that was associated with the underlying Pseudomonas sepsis that may have led to transient deterioration in graft function, which may have supported the development of calcification of the resolving right upper lobe pneumonia.

\section{Conclusions}

We present a case of extensive localized pulmonary calcifications in an immunosuppressed patient with a recent kidney transplant and treatment for a Pseudomonas pneumonia. Given that the localization of the pneumonia appears to overlap with the area of pulmonary calcification, it is most likely that pulmonary calcification is related to the resolving post infectious process. This is classically reported in varicella pneumonia but has yet to be associated with a Pseudomonas infection. Further supporting this, the patient has not experienced any recurrence or involvement of other areas of the lung. Localized pulmonary calcification should be considered as part of the differential diagnosis in patients with persistent opacities following resolution of a pneumonia.

\section{Additional Information}

\section{Disclosures}

Human subjects: Consent was obtained by all participants in this study. Patient consent is waived as this is a pathology case report. All nonrelevant history and demographics have been removed from the case to protect patient identity. Conflicts of interest: In compliance with the ICMJE uniform disclosure form, all authors declare the following: Payment/services info: All authors have declared that no financial support was received from any organization for the submitted work. Financial relationships: All authors have declared that they have no financial relationships at present or within the previous three years with any organizations that might have an interest in the submitted work. Other relationships: All authors have declared that there are no other relationships or activities that could appear to have influenced the submitted work.

\section{Acknowledgements}

Matthew J. Cecchini and Dominic L. Shepherd contributed equally to this work.

\section{References}

1. Bendayan D, Barziv Y, Kramer MR: Pulmonary calcifications: a review. Respir Med. 2000, 94:190-193. 10.1053/rmed.1999.0716

2. Hartman TE, Muller NL, Primack SL, Johkoh T, Takeuchi N, Ikezoe J, Swensen SJ: Metastatic pulmonary calcification in patients with hypercalcemia: findings on chest radiographs and CT scans. AJR Am J Roentgenol. 1994, 162:799-802. 10.2214/ajr.162.4.8140993

3. Jost RG, Sagel SS: Metastatic calcification in the lung apex . AJR Am J Roentgenol. 1979, 133:1188-1190. 10.2214/ajr.133.6.1188

4. Raider L: Calcification in chickenpox pneumonia . Chest. 1971, 60:504-507.

5. Ross PJ, Seaton A, Foreman HM, Morris Evans WH: Pulmonary calcification following smallpox handler's lung. Thorax. 1974, 29:659-665.

6. Jarava C, Martí V, Gurpegui ML, Merello JI, Rdez-Quesada B, Palma A: Pulmonary calcification in chronic dialysis patients. Nephrol Dial Transplant. 1993, 8:673-674.

7. Stewart S, Rassl D: Advances in the understanding and classification of pulmonary hypertension. Histopathology. 2009, 54:104-116. 10.1111/j.1365-2559.2008.03180.x

8. Mandel J, Mark EJ, Hales CA: Pulmonary veno-occlusive disease. Am J Respir Crit Care Med. 


\section{Cureus}

2000, 162:1964-1973. 10.1164/ajrccm.162.5.9912045

9. Bloodworth J, Tomashefski JF: Localised pulmonary metastatic calcification associated with pulmonary artery obstruction. Thorax. 1992, 47:174-178. 10.1136/thx.47.3.174

10. Ando T, Mochizuki Y, Iwata T, et al.: Aggressive pulmonary calcification developed after living donor kidney transplantation in a patient with primary hyperparathyroidism.

Transplant Proc. 2013, 45:2825-2830. 10.1016/j.transproceed.2013.01.071 